\title{
Association between Smartphone Usage and Mental Health in South Korean Adolescents: The 2017 Korea Youth Risk Behavior Web-Based Survey
}

\author{
Hyunjeong Kim, Min-Kyoung Cho, Hyeonyoung Ko, Jung Eun Yoo, Yun-Mi Song* \\ Department of Family Medicine, Samsung Medical Center, Sungkyunkwan University School of Medicine, Seoul, Korea
}

See editorial commentary page on 73

Background: Smartphone usage is indispensably beneficial to people's everyday lives. However, excessive smartphone usage has been associated with physical and mental health problems. This study aimed to evaluate the association of smartphone usage with depressive symptoms, suicidal thoughts, and suicide attempts in Korean adolescents.

Methods: This cross-sectional study was conducted in 54,603 Korean adolescent participants (26,930 male and 27,673 female) in the Korea Youth Risk Behavior Survey in 2017 who reported their smartphone use. We performed multiple logistic regression analysis to evaluate the association of smartphone use with mental health after adjusting for relevant covariates.

Results: Among the participants, $25.6 \%$ of male students and $38.4 \%$ of female students reported using their smartphone for at least 30 hours per week. As time duration of smartphone usage increased, the risk of experiencing depressive symptoms, suicidal thoughts, and suicide attempt tended to increase, with odds ratios ( $95 \%$ confidence interval) of 1.18 (1.10-1.26), 1.18 (1.08-1.29), and 1.34 (1.11-1.60), respectively, for high smartphone usage compared with low smartphone usage. These associations remained significant with only slight change in odds ratios after consideration of problems that may be caused by smartphone usage, such as conflicts with family members or peers, or disturbance in school work.

Conclusion: Smartphone overuse was independently associated with an increased risk of mental health problems, which did not seem to be mediated by the problems caused by smartphone usage.

Keywords: Republic of Korea; Adolescent; Smartphone; Depression; Suicidal Ideation; Suicide, Attempted

Received: July 17, 2018, Revised: November 7, 2018, Accepted: July 17, 2019

*Corresponding Author: Yun-Mi Song https://orcid.org/0000-0001-9232-5563

Tel: +82-2-3410-2442, Fax: +82-2-3414-2832, E-mail: yunmisong@skku.edu 


\section{INTRODUCTION}

The smartphone is a mobile phone that may work as a personal computer. After the first introduction of smartphones in 2009, these devices have rapidly penetrated people's daily lives, and the rate of smartphone usage is briskly increasing worldwide. South Korea ranks as the fourth fastest nation in public smartphone distribution rate, ${ }^{1)}$ and, recently, smartphone addiction is becoming a significant important social problem.

According to a 2017 survey by the Ministry of Science, ICT and Future Planning, tendency toward smartphone addiction is especially evident in teenagers; ${ }^{2)} 15$ of 100 students in primary to high school are addicted to smartphones, and 3.5\% among them are considered risky users with serious disabilities in daily life.

Given the association of a range of health problems with addiction to the internet, ${ }^{3-5)}$ excessive smartphone usage might cause negative effects on people's physical and psychosocial health. However, research on the negative effects of smartphone overuse has been scarce ${ }^{6-10)}$ probably owing to the overwhelming benefits that smartphones provide in people's daily lives, such as convenience, mobility, and connectivity. ${ }^{11,12)}$ Depression could be the most important problem associated with smartphone overuse; the psychopathological model suggests that neurotransmitters related with addiction are also involved in the development of depression. ${ }^{13)}$ The prevalence of depressive disorders in adolescence has been reported to be between $15 \%$ and $20 \% .{ }^{14)}$ Unlike in adults, depression in adolescents tends to be characterized by the frequent occurrence of delinquent behaviors, game addiction, and internet addiction. ${ }^{14,15)}$ Thus, researchers need to examine the relation between depression and smartphone overuse in adolescents.

The present study aimed to investigate the association between smartphone usage time and mental health in Korean adolescents, using data from a nationwide survey that recruits representative samples, after adjusting for covariates.

\section{METHODS}

\section{Study Participants}

We used the data from the 13th Korean Youth Risk Behavior Webbased Survey (KYRBWS) collected in 2017. The KYRBWS is a government-approved annual statistical survey (Statistics Korea, approval no., 117058) conducted by the Korea Centers for Disease Control and Prevention since 2005 to monitor priority health risk behaviors among Korean adolescents. The 2017 survey consisted of 123 questions assessing the demographic characteristics and 15 areas of health-related behaviors. Additional details on sampling methodology and survey procedures are available in the survey introduction.

The target population of the 13th KYRBWS was middle- and highschool students at the time of survey in April 2017. A stratified multistage cluster-sampling design was used for the survey to select the nationally representative samples of middle- and high-school students.
Students participated in the survey voluntarily and completed the anonymous, self-administered, web-based survey during a regular class period. Among the total of 64,991 students from 400 middle schools and 400 high schools, 62,276 students participated in the survey, with a response rate of $96.7 \%$. In the present study, we included 54,603 adolescents $(27,673$ female and 26,930 male) who reported their smartphone use. This study was reviewed and approved by the institutional review board of Samsung Medical Center (SMC 2018-03096). The data used in this study did not include any identifiable personal information. Thus, the institutional review board of Samsung Medical Center did not require the acquisition of informed consent forms for the current study.

\section{Study Variables}

From the information collected by KYRBWS, we used the data on sociodemographic characteristics, smartphone usage pattern, and health status. Smartphone usage time was assessed separately for weekdays and weekends by the following question: "During the weekdays of the last 30 days, for how many hours on average did you use a smartphone in one day?" and "During the weekends of the last 30 days, for how many hours on average did you use a smartphone in one day?" Using the responses to the above two questions, we calculated the average duration of smartphone usage per week. Then, we classified participants into three groups according to the tertile distribution of average smartphone use time per week; low-use group ( $<16$ hours), moderate-use group (16-29 hours), and high-use group ( $\geq 30$ hours).

We also assessed problematic smartphone usage by asking the following questions: "Have you ever experienced conflict with family members because of your smartphone use?" "Have you ever experienced conflict with peers because of your smartphone use?" "Have you ever experienced disturbance in your school work because of smartphone use?"

The main outcome variables in the present study were depressive symptoms, suicide ideation, and suicide attempt. We assessed depressive symptoms using the question "Have you ever felt hopeless or sad

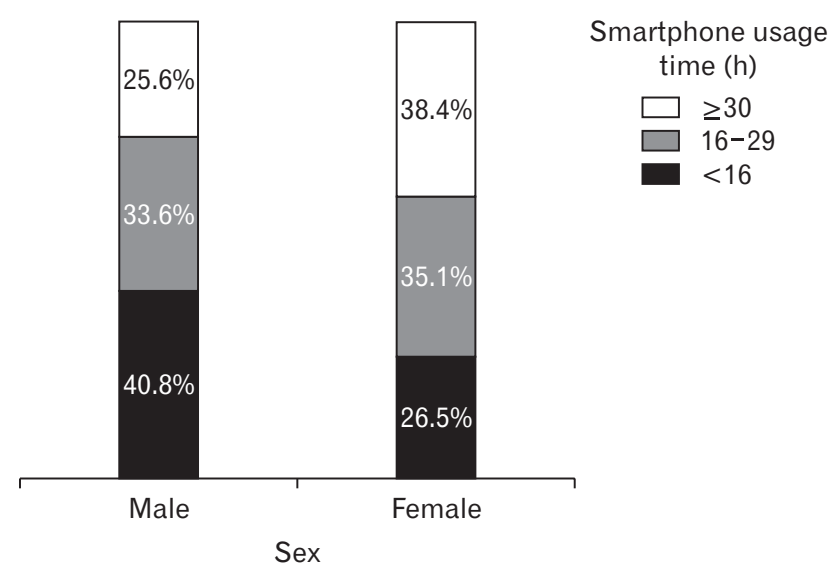

Figure 1. Distribution of smartphone usage time by sex. 
for more than two weeks such that it was difficult to live an ordinary life during the last year?" If a participant answered "yes" to the question, we categorized them in the "depressive symptom group." We assessed suicide ideation and attempt by the questions, "During the last 12 months, have you ever seriously thought about suicide?" and "During the last 12 months, have you ever attempted suicide?" We considered the sociodemographic and health status variables as covariates based on the suggestion of a previous study that these are associated with depression or quality of life in adolescents. ${ }^{14)}$ The sociodemographic and behavioral variables were age, sex, economic status, living arrangement (living apart from family), presence of siblings, experience of school violence, school performance, alcohol drinking, smoking, and physical activity. The health status variables were self-reported health condition, sleep satisfaction, stress level, and well-being.

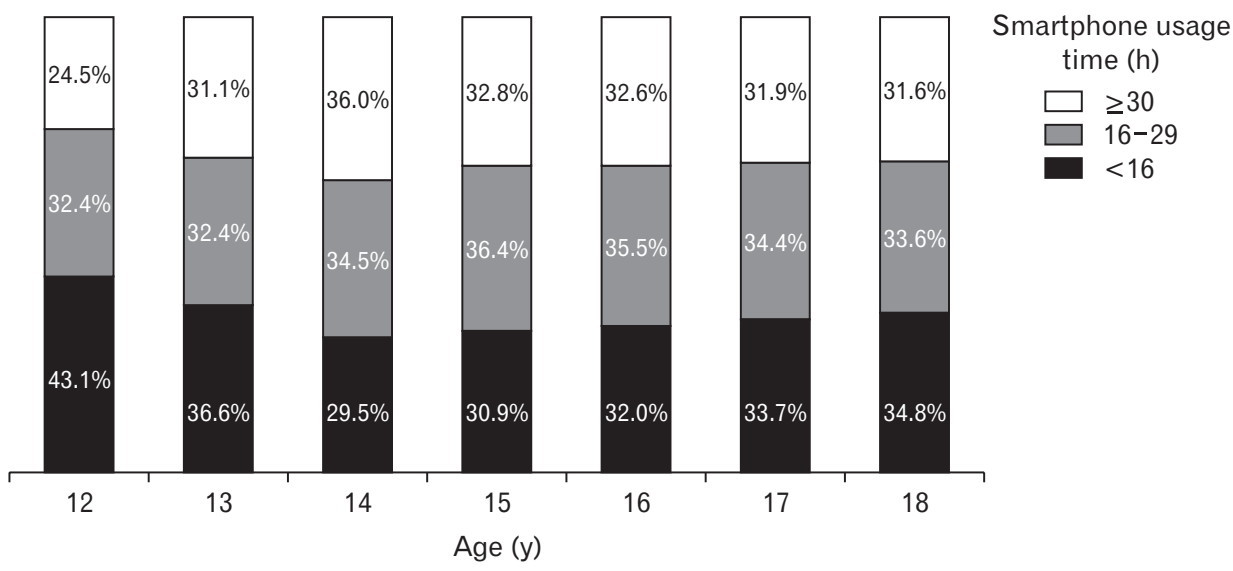

Figure 2. Distribution of smartphone usage time by age.

Table 1. Socioeconomic and behavioral characteristics of participants according to smartphone usage time

\begin{tabular}{|c|c|c|c|c|c|c|}
\hline \multirow{2}{*}{ Variable } & \multirow{2}{*}{ Category } & \multirow{2}{*}{ Overall } & \multicolumn{3}{|c|}{ Smartphone usage time per week } & \multirow{2}{*}{ P-value } \\
\hline & & & Low $(<16$ h) & Moderate (16-29 h) & High ( $\geq 30$ h) & \\
\hline No. of persons & & $54,603(100)$ & $18,338(33.6)$ & $18,731(34.3)$ & $17,534(32.1)$ & \\
\hline Age (y) & & $15.0 \pm 1.75$ & $14.9 \pm 1.82$ & $15.0 \pm 1.73$ & $15.1 \pm 1.70$ & $<0.001$ \\
\hline \multirow[t]{2}{*}{ Sex } & Male & $26,930(50.6)$ & $11,001(60.9)$ & $9,021(49.3)$ & $6,908(40.8)$ & $<0.001$ \\
\hline & Female & $27,673(49.4)$ & $7,337(39.1)$ & $9,710(50.7)$ & $10,626(59.2)$ & \\
\hline \multirow[t]{5}{*}{ Economic status } & High & $5,510(10.3)$ & $2,534(13.9)$ & $1,598(8.7)$ & $1,378(8.1)$ & $<0.001$ \\
\hline & Upper-middle & $16,009(29.7)$ & $6,155(34.2)$ & $5,525(29.6)$ & $4,329(24.8)$ & \\
\hline & Middle & $25,297(46.0)$ & $7,732(41.7)$ & $9,016(48.0)$ & $8,549(48.5)$ & \\
\hline & Low-middle & $6,453(11.6)$ & $1,600(8.6)$ & 2,209 (11.6) & $2,644(15.0)$ & \\
\hline & Low & $1,334(2.4)$ & $317(1.7)$ & $383(2.1)$ & $634(3.7)$ & \\
\hline Living apart from family & & $87(0.2)$ & $30(0.1)$ & $20(0.1)$ & $37(0.2)$ & 0.254 \\
\hline Only child & & $7,197(13.3)$ & $2,669(14.8)$ & $2,315(12.4)$ & $2,213(12.7)$ & $<0.001$ \\
\hline School violence experience & & $1,186(2.2)$ & $434(2.3)$ & $323(1.8)$ & $429(2.5)$ & 0.652 \\
\hline Drinking experience & & 21,897 (41.1) & $5,854(33.0)$ & $7,372(40.5)$ & $8,671(50.6)$ & $<0.001$ \\
\hline Smoking experience & & $7,124(13.7)$ & $1,710(9.9)$ & $2,244(12.7)$ & $3,170(18.9)$ & $<0.001$ \\
\hline \multirow[t]{3}{*}{ Physical activity } & High & $6,761(13.1)$ & $2,439(14.1)$ & $2,284(12.7)$ & $2,038(12.3)$ & $<0.001$ \\
\hline & Moderate & $14,882(29.8)$ & 5,225 (31.5) & $5,131(29.8)$ & $4,526(27.9)$ & \\
\hline & Low & $28,513(57.2)$ & $9,005(54.4)$ & $9,823(57.5)$ & 9,685 (59.8) & \\
\hline \multirow[t]{5}{*}{ School performance } & High & 7,476 (13.5) & $3,726(20.0)$ & $2,355(12.2)$ & $1,395(7.9)$ & $<0.001$ \\
\hline & Upper-middle & $14,437(26.3)$ & $5,718(31.4)$ & 5,177 (27.2) & $3,542(19.6)$ & \\
\hline & Middle & $15,707(28.9)$ & $5,014(27.7))$ & $5,767(31.1)$ & 4,926 (27.9) & \\
\hline & Low-middle & $11,882(21.8)$ & $2,859(15.4)$ & $4,013(21.7)$ & $5,010(28.9)$ & \\
\hline & Low & $5,101(9.4)$ & $1,021(5.5)$ & $1,419(7.8)$ & $2,661(15.7)$ & \\
\hline \multicolumn{7}{|c|}{ Problems caused by smartphone } \\
\hline Conflict with family members & & $12,001(22.3)$ & $3,200(18.0)$ & $4,257(23.0)$ & $4,544(26.3)$ & $<0.001$ \\
\hline Conflict with peers & & $1,899(3.4)$ & $380(2.0)$ & $574(3.1)$ & $945(5.4)$ & $<0.001$ \\
\hline Disturbance in school work & & $13,473(25.8)$ & $3,143(18.2)$ & $5,112(28.7)$ & $5,218(31.2)$ & $<0.001$ \\
\hline
\end{tabular}

Values are presented as number of persons (weighted \%) or mean \pm standard deviation.

${ }^{*}$ P-values for the differences in variables among the three groups by smartphone usage time were obtained by chi-square test for categorical variables or by analysis of variance for numerical variables. 


\section{Statistical Analysis}

All statistical analyses were conducted with consideration of the multistage cluster-sampling design. We performed chi-square tests for categorical variables and Student t-tests for continuous variables to examine the relation between each variable and smartphone usage time.

Odds ratios (ORs) with 95\% confidence intervals (CIs) were estimated by multiple logistic regression analysis to evaluate associations between smartphone usage time and depressive symptoms after adjusting for covariates. The covariates were selected from the findings of univariate analyses in our study and previous studies. ${ }^{6,8,10-12,14-16)}$

We repeated the analysis using an age- and sex-adjusted model and three additional models. In model 1, age, sex, socioeconomic variables (economic status, presence of siblings, and living apart from family) were adjusted. Behavioral characteristics (school violence experience, drinking experience, smoking experience, and physical activity) and self-reported health status (health condition, sleep satisfaction, stress level, and well-being) were additionally adjusted in model 2.

We hypothesized that problems caused by smartphone usage may mediate the association between depressive symptoms and smartphone usage time. Thus, we further adjusted for problems that may be caused by smartphone usage (conflict with family, conflict with peers, and disturbance in school work) in model 3, in addition to the covariates included in model 2.

All statistical analyses were performed using PASW SPSS Statistics ver. 18.0 (SPSS Inc., Chicago, IL, USA). The statistical significance of each result was assessed based on $\mathrm{P}<0.05$.

\section{RESULTS}

The mean ( \pm standard deviation) smartphone usage time per a week was $31.5( \pm 24.2)$ hours in female students and $25.3( \pm 21.9)$ hours in male students. Among the total of 54,603 participants, $25.6 \%$ of the male students and $38.4 \%$ of the female students reported using a smartphone for 30 hours and more (Figure 1). By age group, 14-yearold students had the highest proportion of high smartphone usage (Figure 2).

Table 1 shows the distribution of the sociodemographic and behavioral characteristics, as well as problems caused by smartphone use, according to smartphone usage time. The high-smartphone usage group had a higher proportion of female students compared with the low smartphone-usage group. The proportion of students who reported their economic status and school performance as belonging to the lowest quantile increased with the increase in smartphone usage time. No significant difference was observed in the proportion of students living apart from family or having experienced school violence according to smartphone usage time. The proportions of students with alcohol drinking and smoking experience were higher in the high-smartphone usage group than in the low-use group. Meanwhile, the propor-

Table 2. Self-reported health status of participants according to smartphone usage time

\begin{tabular}{|c|c|c|c|c|c|c|}
\hline \multirow{2}{*}{ Variable } & \multirow{2}{*}{ Category } & \multirow{2}{*}{ Overall } & \multicolumn{3}{|c|}{ Smartphone usage time per week } & \multirow{2}{*}{ P-value* } \\
\hline & & & Low $(<16$ h) & Moderate (16-29 h) & High ( $\geq 30$ h) & \\
\hline \multirow[t]{5}{*}{ Health condition } & Very healthy & $14,771(26.8)$ & $5,787(31.2)$ & $4,821(25.4)$ & $4,163(23.4)$ & \multirow[t]{5}{*}{$<0.001$} \\
\hline & Healthy & $24,438(44.7)$ & $8,271(45.0)$ & $8,700(46.3)$ & $7,467(42.4)$ & \\
\hline & Fair & $11,872(22.0)$ & $3,372(18.8)$ & $4,068(22.0)$ & $4,432(25.5)$ & \\
\hline & Unhealthy & $3,280(6.1)$ & $831(4.6)$ & $1,083(6.0)$ & 1,366 (8.0) & \\
\hline & Very unhealthy & $242(0.5)$ & $77(0.4)$ & $59(0.3)$ & $106(0.7)$ & \\
\hline \multirow[t]{5}{*}{ Sleep satisfaction } & More than enough & $4,169(7.3)$ & $1,736(9.0)$ & $1,261(6.5)$ & $1,172(6.4)$ & \multirow[t]{5}{*}{$<0.001$} \\
\hline & Enough & $9,444(16.8)$ & $3,598(18.9)$ & $3,306(16.9)$ & $2,540(14.3)$ & \\
\hline & Fair & $17,585(32.3)$ & $5,674(30.9)$ & $6,218(33.2)$ & $5,693(31.6)$ & \\
\hline & Not enough & $15,663(29.0)$ & 4,972 (27.9) & $5,461(29.6)$ & $5,230(31.9)$ & \\
\hline & Not nearly enough & $7,742(14.6)$ & $2,358(13.3)$ & 2,485 (13.8) & $2,899(36.1)$ & \\
\hline \multirow[t]{5}{*}{ Stress level } & No & $1,971(3.6)$ & $884(4.6)$ & $577(3.0)$ & $510(3.0)$ & \multirow[t]{5}{*}{$<0.001$} \\
\hline & Low & $8,917(16.2)$ & $3,475(18.5)$ & $3,086(16.4)$ & 2,356 (13.3) & \\
\hline & Middle & $23,129(42.7)$ & $7,930(43.8)$ & $8,202(43.9)$ & $6,997(40.2)$ & \\
\hline & Upper-middle & $15,006(27.5)$ & $4,514(24.7)$ & $5,208(28.0)$ & $5,284(30.0)$ & \\
\hline & High & $5,580(10.1)$ & $1,535(8.4)$ & $1,658(8.7)$ & $2,387(13.5)$ & \\
\hline \multirow[t]{5}{*}{ Well-being } & Very happy & $15,944(28.5)$ & $6,203(32.9)$ & $5,290(27.5)$ & $4,451(24.8)$ & \multirow[t]{5}{*}{$<0.001$} \\
\hline & Happy & $21,013(38.6)$ & 7,049 (38.8) & $7,545(40.3)$ & $6,419(36.6)$ & \\
\hline & Fair & $13,403(25.1)$ & $3,900(21.8)$ & $4,572(25.1)$ & 4,391 (28.6) & \\
\hline & Unhappy & $3,583(6.6)$ & $981(5.4)$ & $1,162(6.2)$ & $1,440(8.3)$ & \\
\hline & Very unhappy & $660(1.2)$ & $205(1.2)$ & $162(0.9)$ & $293(1.7)$ & \\
\hline Depressive symptoms $^{\dagger}$ & Yes & $13,850(25.4)$ & $3,841(21.0)$ & $4,526(24.4)$ & $5,483(31.3)$ & $<0.001$ \\
\hline Suicidal thoughts ${ }^{\dagger}$ & Yes & $6,714(12.2)$ & $1,793(9.8)$ & $2,094(11.1)$ & $2,827(16.0)$ & $<0.001$ \\
\hline Suicide attempts ${ }^{\dagger}$ & Yes & $2,108(3.8)$ & $603(3.3)$ & $608(3.2)$ & $897(5.1)$ & $<0.001$ \\
\hline
\end{tabular}

Vaues are presented as number of persons (weighted \%).

${ }^{*}$ P-values were obtained by chi-square test. 'We defined depressive symptoms as having ever felt hopelessness or grief for more than 2 weeks that negatively affected daily living in the last year; suicidal thoughts as having ever thought about suicide in the last year; suicide attempts as having ever committed a suicide attempt in the last year. 
tion of students who reported engaging in physical activities was higher in the low-use group than in the high-use group. Problems attributed to smartphone usage, including conflict with family members or peers and disturbance in school work, increased with the increase of smartphone usage time.

Table 2 gives the self-reported health status of the participants. The proportion of students who reported their health condition as "very healthy" was significantly higher in the low smartphone-use group compared with the high-use group. Compared with those in the low smartphone-use group, significantly higher proportions of students in the high-smartphone use group reported having felt depressive symptoms for more than two weeks (31.3\% versus $21.0 \%$ ), had a suicidal thought during the last 12 months (16.0\% versus $9.8 \%)$, and attempted suicide during the last 12 months (5.1\% versus $3.3 \%$ ).

Table 3 shows the multivariable-adjusted association of smartphone usage time with depressive symptoms, suicidal thoughts, and suicide attempts. Smartphone usage time was positively associated with depressive symptoms, suicidal thoughts, and suicide attempts in ageand sex-adjusted analyses. After additional adjustment for behavioral characteristics and self-reported health status, the associations were attenuated but persisted ( $\mathrm{P}$ trend $<0.001$ ). Compared with the low smartphone-use group, the moderate (OR, 1.07; 95\% CI, 1.01-1.14) and high smartphone-use groups (OR, 1.18; 95\% CI, 1.10-1.26) had a higher risk of depressive symptoms after adjusting for covariates. The ORs (95\% CI) for suicidal thoughts and suicide attempts associated with high smartphone usage were 1.18 (1.08-1.29) and 1.34 (1.11$1.60)$, respectively.

We further adjusted for problems attributed to smartphone usage to examine whether these problems played a role as mediators in the relations between smartphone usage time and depressive symptoms, suicidal thoughts, and suicide attempts. The associations remained significant with minimal change in ORs. Multivariable-adjusted analy- sis in each sex showed similar findings (Supplementary Table 1).

\section{DISCUSSION}

In this large-scale cross-sectional study conducted in a representative sample of Korean adolescents, we found that smartphone usage time was positively associated with depressive symptoms, suicidal thoughts, and suicide attempts. This association persisted even after adjusting for a wide range of covariates.

The present findings are consistent with previous ones: smartphone or internet overuse is associated with mental health, including depression and anxiety. ${ }^{6,16-18)}$ Several explanations could be hypothesized for the association between excessive smartphone use and mental health problems. First, excessive smartphone use tends to cause sleep disturbance by keeping a person up late, thus inducing stress and depression. ${ }^{6)}$ In the present study, participants in the high-usage group were less likely to have sleep satisfaction. Second, people who use smartphones for a longer time tend to have relatively less interaction with other people and to receive lower social support, compared with those who use their smartphone less. ${ }^{6}$ In accordance with this explanation, we found that a greater proportion of the high smartphone-usage group had a conflict with family members and peers. A lack of communication is known to result in feelings of loneliness and depressive moods in children and adolescents. ${ }^{14)}$ Smartphone usage accompanied by social problems has also been associated with various psychological and behavioral problems, such as depression, anxiety, and sleep disturbance. ${ }^{10,12,19)}$ However, the current finding that the association persisted even after adjusting for communication problems and disturbance in school work does not support the hypothesis that communication problems attributed to smartphone usage fully mediate the association between smartphone usage time and mental health problems. Third, reduced physical activity levels by smartphone us-

Table 3. Multivariable-adjusted association* of smartphone usage time with depressive symptoms, suicidal thoughts, and suicide attempts

\begin{tabular}{|c|c|c|c|c|c|}
\hline & Smartphone usage time & Unadjusted & Model $1^{\dagger}$ & Model $2^{\dagger}$ & Model $3^{\dagger}$ \\
\hline \multirow[t]{4}{*}{ Depressive symptoms } & Low & 1 & 1 & 1 & 1 \\
\hline & Moderate & $1.14(1.09-1.20)$ & $1.13(1.07-1.19)$ & $1.07(1.01-1.14)$ & $1.03(0.97-1.10)$ \\
\hline & High & $1.56(1.41-1.65)$ & $1.52(1.44-1.60)$ & $1.18(1.10-1.26)$ & $1.13(1.06-1.21)$ \\
\hline & $\mathrm{P}_{\text {trend }}{ }^{\ddagger}$ & $<0.001$ & $<0.001$ & $<0.001$ & $<0.001$ \\
\hline \multirow[t]{4}{*}{ Suicidal thoughts } & Low & 1 & 1 & 1 & 1 \\
\hline & Moderate & $1.10(1.02-1.18)$ & $1.07(1.00-1.15)$ & $1.03(0.95-1.12)$ & $1.00(0.92-1.09)$ \\
\hline & High & $1.61(1.50-1.73)$ & $1.52(1.41-1.63)$ & $1.18(1.08-1.29)$ & $1.14(1.05-1.24)$ \\
\hline & $P_{\text {trend }}{ }^{\ddagger}$ & $<0.001$ & $<0.001$ & $<0.001$ & 0.001 \\
\hline \multirow[t]{4}{*}{ Suicide attempts } & Low & 1 & 1 & 1 & 1 \\
\hline & Moderate & $1.12(0.94-1.32)$ & $1.08(0.91-1.28)$ & $0.97(0.80-1.17)$ & $0.96(0.79-1.17)$ \\
\hline & High & $2.03(1.72-2.39)$ & $1.90(1.61-2.25)$ & $1.34(1.11-1.60)$ & $1.32(1.10-1.59)$ \\
\hline & $\mathrm{P}_{\text {trend }}{ }^{\ddagger}$ & $<0.001$ & $<0.001$ & $<0.001$ & $<0.001$ \\
\hline
\end{tabular}

Vaues are presented as odds ratio (95\% confidence interval).

${ }^{*}$ Odds ratios (95\% confidence intervals) were estimated by multiple logistic regression analysis. 'In model 1 , age, sex, economic status, and living apart from family were adjusted. Behavioral characteristics (school violence experience, drinking experience, smoking experience, physical activity, and self-reported health status [health condition, subjective well-being, stress, and sleep satisfaction]) were additionally adjusted in model 2. In model 3, problems caused by smartphone usage (conflict with family, conflict with peers, and disturbance in school work) were adjusted along with the covariates in model 2. f for trend was assessed by multiple logistic regression analysis, in which each category of smartphone usage time was keyed in as a numerical term. 
age, as found in our study, may also cause adverse effects on mental health in adolescents. ${ }^{20)}$ Lastly, to explain the biological mechanism of smartphone addiction, a study assessed the gamma-aminobutyric acid (GABA) and glutamate levels in 10 male teenagers with smartphone addiction and 19 control teenagers without smartphone addiction, using brain magnetic resonance spectroscopy. ${ }^{13)}$ This study found higher GABA level in the smartphone addiction group compared with the control group. ${ }^{13)}$ High GABA levels cause a decline in the control of neuro-signals, including cognitive and emotional control, which in turn causes sleepiness, and even anxiety and depression in serious cases. $^{21)}$

The prevalence of smartphone overuse in Korean adolescents found in our study seemed to be very high; about one-third of the participants reported using a smartphone for 30 hours per week. However, we could not compare the prevalence with that in other populations owing to the lack of community studies in other countries.

In our study, the proportion of smartphone high usage was higher among female students than that among male students. Studies have reported discrepancies in smartphone usage time between the two sexes. ${ }^{7,8,12)}$ Roberts et al. ${ }^{11)}$ found that cellphone addiction is largely driven by a desire to connect socially, such as through voice calls, text messages, and social networks. Regarding the purpose of smartphone usage, men tend to use smartphones to play games, watch videos, or listen to music, whereas women tend to seek social contact, such as through messaging and social networking. ${ }^{6,9)}$ These findings suggest that women are more likely to be addicted to smartphone use compared with men.

Our study is the first large-scale research that investigated the association between smartphone usage time and depressive symptoms in Korean adolescents using a nationally representative sample. Moreover, we took into consideration a wide range of covariates that may have influenced depressive symptoms, including clinical conditions.

However, our study has a number of limitations. First, owing to the cross-sectional study design, we were unable to identify the causal relation between smartphone usage time and depressive symptoms. Second, we could not make definite diagnoses of depressive disorders because the data lacked the detailed questions needed for diagnosing clinical depression. Third, the use of data from self-reported questionnaires in analyses might have introduced recall bias. Fourth, we could not consider some covariates that probably influence the smartphone usage time of adolescents, such as time spent alone at home and parents' employment status. Finally, we could not define the smartphone addiction group as in other studies because no questionnaire measuring smartphone addiction was included in the KYRBWS. ${ }^{6}$ )

In conclusion, this study on Korean adolescents showed that smartphone usage time was associated with mental health problems, such as depressive symptoms, suicidal thoughts, and suicide attempts, and the associations did not seem to be mediated by the problems attributed to smartphone usage. Targeted strategies are needed to reduce the negative mental health problems in adolescents who use their smartphone excessively.

\section{CONFLICT OF INTEREST}

No potential conflict of interest relevant to this article was reported.

\section{ACKNOWLEDGMENTS}

This study was supported by a grant from the National R\&D Program for Cancer Control, Ministry of Health and Welfare, Republic of Korea (1720270).

\section{SUPPLEMENTARY MATERIALS}

Supplementary materials can be found via https://doi.org/10.4082/ kjfm.18.0108. Supplementary Table 1. Multivariable-adjusted association of smartphone usage time with depressive symptoms, suicidal thoughts, and suicide attempts in male and female participants.

\section{ORCID}

Hyunjeong Kim: https://orcid.org/0000-0002-8288-115X

Min-Kyoung Cho: https://orcid.org/0000-0001-9453-3366

Hyeonyoung Ko: https://orcid.org/0000-0001-6064-8931

Jung Eun Yoo: https://orcid.org/0000-0001-8344-6291

Yun-Mi Song: https://orcid.org/0000-0001-9232-5563

\section{REFERENCES}

1. Top 50 countries by smartphone users and penetration. Amsterdam: Newzoo; 2017.

2. Smartphone dependency survey. Seoul: Ministry of Science, ICT and Future Planning; 2017.

3. Gamez-Guadix M, Calvete E, Orue I, Las Hayas C. Problematic Internet use and problematic alcohol use from the cognitive-behavioral model: a longitudinal study among adolescents. Addict Behav 2015; 40:109-14.

4. Lam LT. Risk factors of Internet addiction and the health effect of internet addiction on adolescents: a systematic review of longitudinal and prospective studies. Curr Psychiatry Rep 2014;16:508.

5. Yoo YS, Cho OH, Cha KS. Associations between overuse of the internet and mental health in adolescents. Nurs Health Sci 2014;16:193-200.

6. Chen B, Liu F, Ding S, Ying X, Wang L, Wen Y. Gender differences in factors associated with smartphone addiction: a cross-sectional study among medical college students. BMC Psychiatry 2017;17:341.

7. Demirci K, Akgonul M, Akpinar A. Relationship of smartphone use severity with sleep quality, depression, and anxiety in university students. J Behav Addict 2015;4:85-92.

8. Kim SE, Kim JW, Jee YS. Relationship between smartphone addiction and physical activity in Chinese international students in Korea. J Behav Addict 2015;4:200-5.

9. Liu CH, Lin SH, Pan YC, Lin YH. Smartphone gaming and frequent use pattern associated with smartphone addiction. Medicine (Baltimore) 2016;95:e4068.

10. Wolniewicz CA, Tiamiyu MF, Weeks JW, Elhai JD. Problematic smart- 
phone use and relations with negative affect, fear of missing out, and fear of negative and positive evaluation. Psychiatry Res 2018;262:61823.

11. Roberts JA, Yaya LH, Manolis C. The invisible addiction: cell-phone activities and addiction among male and female college students. J Behav Addict 2014;3:254-65.

12. De-Sola Gutierrez J, Rodriguez de Fonseca F, Rubio G. Cell-phone addiction: a review. Front Psychiatry 2016;7:175.

13. Seo HS, Jeong EK, Choi S, Kwon Y, Park HJ, Kim I. Neurotransmitters in young people with internet and smartphone addiction: a comparision with normal controls and changes after cognitive behavioral therapy. Oak Brook (IL): Radiological Society of North America; 2017.

14. Birmaher B, Ryan ND, Williamson DE, Brent DA, Kaufman J, Dahl RE, et al. Childhood and adolescent depression: a review of the past 10 years. Part I. J Am Acad Child Adolesc Psychiatry 1996;35:1427-39.

15. Hazell P. Depression in children and adolescents. BMJ Clin Evid 2011;2011.

16. Park S, Jeon HJ, Bae JN, Seong SJ, Hong JP. Prevalence and psychiatric comorbidities of internet addiction in a nationwide sample of Korean adults. Psychiatry Investig 2017;14:879-82.

17. Kim K, Ryu E, Chon MY, Yeun EJ, Choi SY, Seo JS, et al. Internet addiction in Korean adolescents and its relation to depression and suicidal ideation: a questionnaire survey. Int J Nurs Stud 2006;43:185-92.

18. Sohn M, Oh H, Lee SK, Potenza MN. Suicidal ideation and related factors among Korean high school students: a focus on cyber addiction and school bullying. J Sch Nurs 2018;34:310-8.

19. Thomee S, Harenstam A, Hagberg M. Mobile phone use and stress, sleep disturbances, and symptoms of depression among young adults: a prospective cohort study. BMC Public Health 2011;11:66.

20. Schuch FB, Vancampfort D, Firth J, Rosenbaum S, Ward PB, Silva ES, et al. Physical activity and incident depression: a meta-analysis of prospective cohort studies. Am J Psychiatry 2018;175:631-48.

21. Lener MS, Niciu MJ, Ballard ED, Park M, Park LT, Nugent AC, et al. Glutamate and gamma-aminobutyric acid systems in the pathophysiology of major depression and antidepressant response to ketamine. Biol Psychiatry 2017;81:886-97. 